\title{
The potential of irisin as a therapeutic for diabetes
}

\author{
"Irisin was identified in 2012 and shown to be a novel metabolic \\ regulator since then; subsequent information on the potential roles \\ of irisin in regulating hepatic and pancreatic islet functions remains \\ elusive."
}

First draft submitted: 13 February 2017; Accepted for publication: 20 February 2017; Published online: 12 April 2017

Keywords: adipose $\bullet$ AMPK $\bullet$ exercise $\bullet$ FNDC5 $\bullet$ insulin resistance $\bullet$ irisin $\bullet$ liver - myokine $\bullet$ obesity $\bullet$ pancreatic islet $\bullet$ skeletal muscle

Diabetes and obesity-related diseases are a major drain on healthcare resources; it is reported that around 350 million people suffer from diabetes globally, being Type 2 diabetes mellitus (T2DM) the most prevalent [1]. Given that current remedies for diabetes are suboptimal, it is of paramount importance to explore some novel therapeutic targets as well as to promote preventative measures, such as dietary- and exerciserelated approaches. T2DM develops once there are increases in peripheral insulin resistance, which is accompanied with pancreatic islet demise. In this regard, dysregulation of hepatic metabolism and islet function contributes critically to insulin resistance and insulin insufficiency, respectively, the major risk factors for the progression to the pathogenesis of T2DM; therefore, preservation of the liver and islet functions is a key strategy for the management of T2DM.

The benefits of exercise on cardiometabolic syndromes are well recognized. Skeletal muscle has been reported as an endocrine organ while exercise-induced secretion of small protein molecules, termed myokines, mediates its demonstrated actions [2]. In this context, irisin is a newly identified myokine, which is demonstrated to exhibit therapeutic potential in obesity and T2DM, stimulating 'browning' of white adipose tissue [3]. Subsequent studies have shown that irisin promotes glucose uptake in skeletal muscle while improving hepatic glucose and lipid metabolism, thus probably attributing to the alleviation of hyperlipidemia and hyperglycemia in obesity-related metabolic disease [4]. These findings point to irisin as a potent metabolic player in T2DM-relevant organs, such as the liver and pancreas; however, the proposed mechanism(s) whereby irisin regulates hepatic metabolism and modulates islet function remains an intriguing and unresolved question. Against this background, it prompts us to postulate that irisin can improve hepatic glucose and lipid metabolism through modulating reductions in endoplasmic reticulum (ER) stress, as well as promoting islet $\beta$-cell function and survival, thereby ameliorating hyperglycemia, hyperlipidemia and insulin resistance. If confirmed, irisin might simultaneously reduce these abnormalities of hepatic and islet functions that are believed to effectively combat T2DM risk.

The liver helps maintain normal glucose homeostasis by striking a balance between hepatic glucose output (gluconeogenesis and glycogenolysis) and glucose storage (glycogenesis). Rates of gluconeogenesis are primarily determined by the transcriptional levels of two gluconeogenic enzyme genes, phosphoenolpyruvate carboxykinase (Pepck) and glucose-6-phosphatase (G-6-pase); glycogenesis is modulated by the activity of GSK-3 and glycogen synthase, while inhibition of GSK-3 promotes glycogen synthase activity and glycogen synthesis [5]. Being able to be a direct regulation of glucose metab-

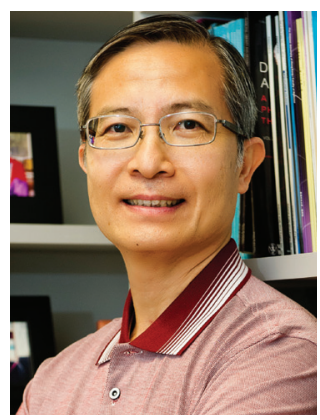

Po Sing Leung

Lo Kwee-Seong Integrated Biomedical Sciences Building, School of Biomedical Sciences, Faculty of Medicine, The Chinese University of Hong Kong, Shatin, New Territories, Hong Kong, PR China

Tel.: +85239436879

Fax: +852 26035123

psleung@cuhk.edu.hk 
olism, hepatic lipid metabolism has a critical role in glucose homeostasis as well. Carbohydrate is converted to fat by de novo lipogenesis when its intake exceeds storage and oxidation capacities, but excessive hepatic lipid causes inflammation and hepatic insulin resistance [6]. Considerable evidences have suggested that ER stress is closely associated with metabolic disorders; in particular, hepatic ER stress promotes hepatic glucose production, lipogenesis and insulin resistance in obese and diabetic conditions and, therefore, maintenance of normality in hepatic cellular metabolic function is indispensable for preventing hepatic insulin resistance and T2DM [7]. Activation of hepatic AMPK exerts antidiabetic actions through modulation of hepatic glucose and lipid metabolism with attenuation of lipogenesis and gluconeogenesis, as well as promotion of lipid oxidation and glycolysis [8]. Interestingly, a recent study has demonstrated that irisin also activates AMPK in skeletal muscle [9]. Whether irisin regulates the hepatic AMPK-Foxo1 signaling pathways and thus ameliorates hepatic ER stress as well as glucose and lipid metabolism require investigations.

\section{"Intriguingly, a more recent study has demonstrated that irisin activates the Akt signaling pathway in myocardial cells."}

Emerging data from physiological and genetic studies have shown that islet dysfunction and loss of $\beta$-cell mass are the key determinants of whether an insulinresistant state will progress to frank hyperglycemia and diabetes, since insulin resistance alone is insufficient for the development of T2DM [10]. High circulating concentrations of glucose and fatty acids in diabetic states are attributed to progressive loss of islet function and reduced islet survival through mechanisms that are involved in increased oxidative ER stress and inflammation, a process called glucolipotoxicity. In view of this, agents or factors that protect islets against glucolipotoxicity are potentially valuable for the management of T2DM. Several signaling pathways have been reported to have critical roles in insulin secretion as well as $\beta$-cell growth and survival; for example, activation of Akt is closely associated with $\beta$-cell survival and is involved in the mechanism of compensatory $\beta$-cell growth in the insulin-resistant state [11]. In addition, prior studies have reported that fatty acid induced ER stress in islets is associated with decreased Akt activity and with activation of c-Jun $\mathrm{NH}_{2}$-terminal kinase (JNK), which contribute to $\beta$-cell apoptosis [12]. All of these study findings lend support for Akt as a promising candidate in the preservation of islet function and cell mass. Intriguingly, a more recent study has demonstrated that irisin activates the Akt signaling pathway in myocardial cells [13]. It is thus plausible to posit that irisin also activates the Akt signaling pathway in pancreatic islets while exerting protective effects under diabetic conditions.

Irisin is predominantly produced and secreted from skeletal muscle as myokine [3]; it has been reported that the exercise-induced transcriptional coactivator PGC- $1 \alpha$ stimulates the expression of the fibronectin type III domain containing 5 (FNDC5), which is then cleaved to form irisin, thus elevating the serum levels of irisin $[3,14]$. Secreted irisin is able to promote the browning of white adipocytes and glucose uptake in skeletal muscle [15]. Of note, two independent studies have demonstrated that irisin attenuates palmitic acid induced lipogenesis and glucosamine-induced gluconeogenesis via the mediation of the PRMT3 and PI3K/Akt pathways, respectively, in the hepatocytes [16,17]. Moreover, overexpression of FNDC5/irisin enhances energy expenditure and insulin sensitivity, while reducing hyperglycemia, hyperlipidemia and blood pressure in mice [18]. In addition, a clinical study has reported that serum irisin level was reduced in obese adults with nonalcoholic fatty liver disease, which was directly linked to hepatic triglyceride content [19]. Undoubtedly, both the liver and pancreatic islets have critical roles for the pathogenesis of T2DM and both are susceptible to glucolipotoxicity. Despite these findings, the precise mechanism(s) by which irisin regulates hepatic glucose and lipid homeostasis as well as provides protection against islet dysfunction and demise remains to be fully explored.

In order to fill the mechanistic gap in the neglected area of the potential roles of irisin in hepatic and islet metabolism, it is worthwhile exploring the regulatory actions of irisin in the determination of hepatic ER stress and glucose/lipid metabolism, as well as in the determination of pancreatic islet cell function and survival under diabetic conditions. In term of hepatic function, our most recent findings have demonstrated the involvement of the LKB1/AMPK pathway in the modulatory actions of irisin in the metabolism and cell survival in the human liver cells, HepG2, in an insulinresistant state; irisin ameliorated hepatic insulin resistance, dysregulated glucose output and storage, lipid accumulation and cell death induced by chronic exposure to high-glucose plus high-insulin conditions, a cellular model of hyperglycemia and hyperinsulinemia, mimicking T2DM, in a manner that required AMPK activation [20].

On the other hand, administration of irisin phosphorylated Akt and Foxol in isolated islets; in addition, high glucose plus palmitic acid significantly inhibited glucose-stimulated insulin secretion and induced COX2 expression, a biomarker of inflammation in islets, which were rescued by iri- 
sin (Leung ET AL., Unpublished DATA). All of these data prompt us to speculate on irisin having potential roles in modulating hepatic cell metabolism, as well as potentially modulating islet survival and function. Apart from the skeletal muscle derived source of irisin, we also detected the expression of FNDC5, a gene encoding irisin, in the mouse liver, human HepG2 cells, isolated mouse islet and mouse MIN6 $\beta$ cells; interestingly, treatments with high glucose plus palmitic acid reduced islet FNDC5 mRNA level, while treatments with high-glucose plus high-insulin conditions increased FNDC5 mRNA level in HepG2 cells (Leung ET AL., Unpublished DATA). Together, these data suggest differential roles for liver- and isletderived irisin under stress conditions. Whether liverand islet-derived irisin exert protective effects via the modulation of local metabolism and function under diabetic conditions remain unexplored.

Irisin was identified in 2012 and shown to be a novel metabolic regulator since then [3]; subsequent information on the potential roles of irisin in regulating hepatic and pancreatic islet functions remains elusive. Demonstration of these findings would provide solid evidence that supports irisin as an exer-

\section{References}

1 Danaei G, Finucane MM, Lu Y et al. National, regional, and global trends in fasting plasma glucose and diabetes prevalence since 1980: systematic analysis of health examination surveys and epidemiological studies with 370 country-years and 2.7 million participants. Lancet 378, 31-40 (2011).

2 Pedersen BK, Febbraio MA. Muscles, exercise and obesity: skeletal muscle as a secretory organ. Nat. Rev. 8, 457-465 (2012).

3 Boström P, Wu J, Jedrychowske MP et al. A PGC1- $\alpha-$ dependent myokine that drives brown-fat-like development of white fat and thermogenesis. Nature 481, 463-468 (2012).

4 Chen N, Li Q, Liu J, Jia S. Irisin, an exercise-induced myokine as a metabolic regulator: an update narrative review. Diabetes Metab. Res. Rev. 32, 51-59 (2016).

5 Roach PJ, Cao Y, Corbett CA et al. Glycogen metabolism and signal transduction in mammals and yeast. Adv. Enzyme Regul. 31, 101-120 (1991).

6 Gregor MF, Hotamisligil GS. Inflammatory mechanisms in obesity. Ann. Rev. Immunol. 29, 415-445 (2011).

7 Hotamisligil GS. Endoplasmic reticulum stress and the inflammatory basis of metabolic disease. Cell 40, 900-917 (2010).

8 Long YC, Zierath JR. AMP-activated protein kinase signaling in metabolic regulation. J. Clin. Invest. 116, 1776-1783 (2006).

9 Lee HJ, Lee JO, Kim N et al. Irisin, a novel myokine, regulates glucose uptake in skeletal muscle cells via AMPK. Mol. Endocrinol. 29, 873-881 (2015). cise-induced metabolic mediator for the protection against obesity/diabetes-related metabolic disorders, and that mediates the benefits of promoting exercise as a cost-effective way to reduce the risks of metabolic syndromes. Given the high worldwide prevalence of obesity, T2DM and the related increases in cardiometabolic sequela as well as the resultant costs and socioeconomic burdens, these study findings would provide an avenue for irisin to be developed as a promising preventative and therapeutic agent, which is cost-effective for the management of obesity-related insulin resistance and diabetes.

\section{Financial \& competing interests disclosure}

The work described in this paper was supported by the General Research Fund from the Research Grants Council of the Hong Kong Special Administrative Region, China (Ref. No.: 2140941), awarded to PS Leung. The author has no other relevant affiliations or financial involvement with any organization or entity with a financial interest in or financial conflict with the subject matter or materials discussed in the manuscript apart from those disclosed.

No writing assistance was utilized in the production of this manuscript.

10 Bell GI, Polonsky KS. Diabetes mellitus and genetically programmed defects in beta-cell function. Nature 414, 788-791 (2001).

11 Jetton TL, Lausier J, LaRock K et al. Mechanisms of compensatory $\beta$-cell growth in insulin-resistant rats: roles of Akt kinase. Diabetes 54, 2294-2304 (2005).

12 Srinivasan S, Ohsugi M, Liu ZG, Fatrai S, Mizrachi EB, Permutt MA. Endoplasmic reticulum stress-induced apoptosis is partly mediated by reduced insulin signaling through phosphatidylinositol 3-kinase/Akt and increased glycogen synthase kinase-3 $\beta$ in mouse insulinoma cells. Diabetes 54, 968-975 (2005).

13 Xie C, Zhang Y, Tran TD et al. Irisin controls growth, intracellular $\mathrm{Ca} 2{ }^{+}$signals, and mitochondrial thermogenesis in cardiomyoblasts. PLoS ONE 10, e0136816 (2015).

14 Huh JY, Panagiotou G, Mougios V et al. FNDC5 and irisin in humans: I. Predictors of circulating concentrations in serum and plasma and II. mRNA expression and circulating concentrations in response to weight loss and exercise. Metabolism 61, 1725-1738 (2012).

15 Zhang Y, Li R, Meng Y et al. Irisin stimulates browning of white adipocytes through mitogen-activated protein kinase 38 MAP kinase and ERK MAP kinase signalling. Diabetes 63, 514-525 (2014).

16 Park MJ, Kim DI, Choi JH, Heo YR, Park SH. New role of irisin in hepatocytes: the protective effect of hepatic steatosis in vitro. Cell Signal. 27, 1831-1839 (2015).

17 Liu TY, Shi CX, Gao R et al. Irisin inhibits hepatic gluconeogenesis and increases glycogen synthesis via the 
PI3K/Akt pathway in Type 2 diabetic mice and hepatocytes. Clin. Sci. 129, 839-850 (2015).

18 Xiong XQ, Chen D, Sun HJ, Ding L, Wang JJ, Chen Q et al. FNDC5 overexpression and irisin ameliorate glucose/lipid metabolic derangements and enhance lipolysis in obesity. Biochim. Biophys. Acta 1852, 1867-1875 (2015).
19 Zhang HJ, Zhang XF, Ma ZM et al. Irisin is inversely associated with intrahepatic triglyceride contents in obese adults. J. Hepatol. 59, 557-562 (2013).

20 So WY, Leung PS. Irisin protects hepatic metabolism and survival in insulin-resistant human HepG2 cells through AMP-activated protein kinase signaling. Int. J. Biochem. Cell Biol. 78, 237-247 (2016). 